Jean-Francois Dhainaut

Massimo Antonelli

Patrick Wright

Arnaud Desachy

Jean Reignier

Sylvain Lavoue

Julien Charpentier

Mark Belger

Michael Cobas-Meyer

Cornelia Maier

Mariano A. Mignini

Jonathan Janes

\section{Extended drotrecogin alfa (activated) treatment in patients with prolonged septic shock}

Published online: 14 May 2009

(C) Springer-Verlag 2009

The online version of the original article can be found under doi:10.1007/s00134-009-1436-1.

Electronic supplementary material The online version of this article (doi:10.1007/s00134-009-1502-8) contains supplementary material, which is available to authorized users.

J.-F. Dhainaut · J. Charpentier

Hôpital Cochin, Université Paris Descartes, 27 Rue du Faubourg Saint Jacques, 75679 Paris Cedex 14, France

M. Antonelli

Policlinico Universitario Agostino Gemelli, Largo Agostino

Gemelli, 168 Rome, Italy

P. Wright

Piedmont Respiratory Research Foundation, Moses Cone Hospital, 1200 N Elm St, Greensboro 27401, USA

A. Desachy

CH d'Angoulême Hôpital Girac, 16470 Saint Michel, France

J. Reignier

C.H.D. Les Oudairies, 85925 La Roche Sur Yon Cedex 9, France

S. Lavoue

CHU de Rennes Pontchaillou, 2 rue Henri Le Guilloux,

35033 Rennes, France

M. Belger · J. Janes

Lilly UK, Erl Wood Manor, Windlesham, Surrey GU20 6PH, UK

M. Cobas-Meyer

Lilly Corporate Center, Indianapolis, IN 46285, USA

C. Maier

Lilly Deutschland GmbH, Speciality Care Europe,

Werner-Reimers-Strasse 2-4, 61352 Bad Homburg, Germany
M. A. Mignini

Eli Lilly Italia Spa, Via A Gramsci 731, 50019 Sesto Fiorentino, Italy

Present Address:

J.-F. Dhainaut (汭)

Agence d'évaluation de la recherche et de l'enseignement supérieur, AERES, 20 rue Vivienne, 75002 Paris, France e-mail: dhainaut@aeres-evaluation.fr

Tel.: +33-1-55556045

Fax: $+33-1-55556394$

\section{Erratum to: Intensive Care Med}

\section{DOI 10.1007/s00134-009-1436-1}

Unfortunately this article contained neither the list of primary investigators and participating centres nor a link to the electronic supplementary material.

These errors are rectified here.

\section{EVBQ: list of primary investigators}

\author{
Austria A. Werba \\ AKH - Wien, Universitätsklinik \\ Vienna, Austria \\ K. Laczika \\ AKH - Wien, Universitätsklinik \\ Vienna, Austria \\ Belgium H. Spapen \\ Akademisch Ziekenhuis, VUB Jette \\ Brussels, Belgium \\ T. Dugernier \\ Clinique Saint Pierre \\ Ottignies, Belgium \\ P. F. Laterre \\ Cliniques Universitaires Saint-Luc \\ Brussels, Belgium
}


J.-L. Vincent

Universite Libre De Bruxelles

Brussels, Belgium

J. Decruyenaere

Universitaire Ziekenhuis Gent

Gent, Belgium

J. Raemakers

Sint Augustinus,

GZA Ziekenhuisen

Wilrijk, Belgium

France J.-P. Mira

Hopital Cochin

Paris, France

D. Perrotin

Chru De Tours

Tours, France

P. E. Bollaert

Chu Nancy Hopital Central

Nancy, France

B. François

Réanimation/CIC Inserm 0801

Chru De Limoges Hopital Dupuytren

Limoges, France

J. Reignier

Centre Hospitalier Departemental

La Roche Sur Yon, France

A. Jaeger

Hopital de Hautepierre

Strasbourg, France

J.-P. Sollet

Centre Hospitalier Victor Dupouy

Argenteuil, France

B. Regnier

Groupe Hospitalier Bichat C Bernard

Paris, France

D. Annane

Hopital Raymond Pointcare

Garches, France

R. Thomas

Hopital De Pontchaillou

Rennes, France

J. Durand-Gasselin

Hopital Font-Pre

Toulon, France

G. Bonmarchand

Chu De Rouen Hopital Charles Nicolle Rouen, France

M. Ferrandiere

Hopital Trousseau

Tours, France

A. Desachy

Ch D'Angouleme

Angouleme, France

Germany F. Wyrembek

Evanglisch-Freikirchliches

Krankenhaus und

Herzzentrum

Bernau, Germany

R. Larsen

Universitätsklinikum des Saarlandes

Homburg/Saar, Germany
F. Fiedler

Klinikum Mannheim Fak. F. Klin. Med.

Mannheim der Universitäts

Heidelberg

Mannheim, Germany

M. Gruendling

Klinikum der Ernst-Moritz-Arndt Universität

Greifswald

Greifswald, Germany

W. Reichert

Klinikum Hoyerswerda

Hoyerswerda, Germany

M. Max

Klinikum der Philipps-Universität Marburg/Lahn

Marburg, Germany

Italy

M. Langer

IRCCS Policlinico S. Matteo

Pavia, Italy

U. Buoncristiano

Ospedale Santa Maria Annunziata

Firenze, Italy

A. Peris

Policlinico di Careggi

Firenze, Italy

R. Tetamo

Ospedale Civico E. Benfratelli

Palermo, Italy

M. Ranieri

Azienda Ospedaliera S. Giovanni Battista

Torino, Italy

M. Girardis

Policlinico Universitario Modena

Modena, Italy

M. Antonelli

Policlinico Universitario Agostino Gemelli

Roma, Italy

Poland

E. Mayzner-Zawadzka

Szpital Kliniczny Dzieciatka Jezus,

Katedra i Klinika Anestezjologii I Intensywnej Terapii WUM

Warszawa, Poland

A. Kubler

Katedra I Klinika Intensywnej Terapii Akademii

Medycznej we Wroclawiu

Wroclaw, Poland

W. Gaszynski

Katedra I Klinika Intensywnej Terapii

UM w Lodzi

Lodz, Poland

Spain

M. Jimenez Lendinez

Hospital La Paz

Madrid, Spain

M. V. De La Torre Prados

Hospital Virgen De La Victoria

Malaga, Spain

F. Perez-Cerda/J. Montejo

Hospital 12 De Octubre

Madrid, Spain

J. Caturla Such

Hospital Gral. Universitario De Alicante

Alicante, Spain 


\author{
United J. Eddlestone \\ Kingdom Manchester Royal Infirmary \\ Manchester, UK \\ S. Fletcher \\ Norfolk and Norwich Hospital \\ Norwich, UK \\ A. Davidson \\ Victoria Infirmary \\ Glasgow, UK \\ M. Brockway \\ St. Johns Hospital \\ Livingston, UK \\ C. Mcallister \\ Craigavon Area Hospital \\ Craigavon, Northern Ireland, UK \\ G. Findlay \\ University Hospital of Wales \\ Cardiff, UK \\ J. Trinder \\ Ulster Hospital \\ Belfast, Northern Ireland, UK \\ F. Gao \\ Heart of England NHS Foundation Trust \\ Birmingham, UK \\ United \\ S. Knoper \\ States \\ University of Arizona Col of Medicine \\ Tucson, United States \\ A. H. Morris \\ Lds Hospital \\ Salt Lake City, United States \\ M. B. Rodricks \\ Southeastern Clinical Research \\ Consultants \\ Orlando, United States
}

R. Riker

Maine Medical Center

Portland, United States

J. Steingrub

Baystate Medical Center

Springfield, United States

P. E. Wright

Le Bauer Cardiovascular Research Foundation

Greensboro, United States

C. C. Naum

Respiratory and Critical Care Consultants, P.C. Indianapolis, United States

J. G. Southard

Jennie Edmundson Hospital

Council Bluffs, United States

D. R. Graham

Springfield Clinic Research

Springfield, United States

M. G. Seneff

George Washington University Medical Center

Washington, United States

D. L. Herr

Washington Hospital Center

Washington, United States

K. Krell

Eastern Idaho Medical Consultants

Idaho Falls, United States

B. Friedman

Joseph M. Still Research Foundation Inc.

Augusta, United States

K. van Gundy

University Medical Center

Fresno, United States 